QUARTERLY OF APPLIED MATHEMATICS

VOLUME LXVI, NUMBER 4

DECEMBER 2008, PAGES 707-724

S 0033-569X(08)01121-2

Article electronically published on October 7, 2008

\title{
ASYMPTOTIC BEHAVIOR OF DISCONTINUOUS SOLUTIONS IN 3-D THERMOELASTICITY WITH SECOND SOUND
}

\author{
BY \\ REINHARD RACKE (Department of Mathematics and Statistics, University of Konstanz, 78457 \\ Konstanz, Germany) \\ AND
}

YA-GUANG WANG (Department of Mathematics, Shanghai Jiao Tong University, Shanghai 200240, People's Republic of China)

\begin{abstract}
This paper is devoted to the study of the Cauchy problem for linear and semilinear thermoelastic systems with second sound in three space dimensions with discontinuous initial data. Due to Cattaneo's law, replacing Fourier's law for heat conduction, the thermoelastic system with second sound is hyperbolic. We investigate the behavior of discontinuous solutions as the relaxation parameter tends to zero, which corresponds to a formal convergence of the system to the hyperbolic-parabolic type of classical thermoelasticity. By studying expansions with respect to the relaxation parameter of the jumps of the potential part of the system on the evolving characteristic surfaces, we obtain that the jump of the temperature goes to zero while the jumps of the heat flux and the gradient of the potential part of the elastic wave are propagated along the characteristic curves of the elastic fields when the relaxation parameter goes to zero. An interesting phenomenon is when time goes to infinity: the behavior will depend on the mean curvature of the initial surface of discontinuity. These jumps decay exponentially when time goes to infinity, more rapidly for a smaller heat conductive coefficient in linear problems and in nonlinear problems when certain growth conditions are imposed on the nonlinear functions.
\end{abstract}

Received May 20, 2007.

2000 Mathematics Subject Classification. Primary 35B40, 74F05.

Key words and phrases. Hyperbolic thermoelasticity, semilinear, discontinuous solutions, asymptotic behavior, curvature.

E-mail address: reinhard.racke@uni-konstanz.de

E-mail address: ygwang@sjtu.edu.cn

(C)2008 Brown University Reverts to public domain 28 years from publication 
1. Introduction. Consider the following system of semilinear thermoelasticity with second sound in three space dimensions:

$$
\begin{aligned}
u_{t t}-\mu \Delta u-(\mu+\lambda) \nabla \operatorname{div} u+\beta \nabla \theta & =f\left(u_{t}, \theta\right), \\
\theta_{t}+\gamma \operatorname{div} q+\delta \operatorname{div} u_{t} & =g\left(u_{t}, \theta\right), \\
\tau q_{t}+q+\kappa \nabla \theta & =0,
\end{aligned}
$$

where $u=u(t, x) \in \mathbb{R}^{3}, \theta=\theta(t, x) \in \mathbb{R}, q=q(t, x) \in \mathbb{R}^{3}$ represent the elastic displacement, the temperature and the heat flux, respectively, and are functions of $(t, x) \in$ $(0, \infty) \times \mathbb{R}^{3}$. The parameters $\mu, \lambda, \beta, \gamma, \delta, \tau, \kappa$ are constants satisfying

$$
\mu, \gamma, \tau, \kappa>0,2 \mu+3 \lambda>0, \beta \delta>0
$$

cp. 2, 8, 9, The given functions $f$ and $g$ are assumed to be smooth. For the differential equations (1.1)-(1.3), we impose the following initial conditions:

$$
u(t=0)=u^{0}, u_{t}(t=0)=u^{1}, \theta(t=0)=\theta^{0}, q(t=0)=q^{0} .
$$

We investigate the asymptotic behavior of solutions to (1.1)-(1.4) for which $\left(\nabla u^{0}, u^{1}, \theta^{0}, q^{0}\right)$ may have jumps on an initial surface $\sigma$ given by

$$
\sigma=\left\{x \in \Omega_{0} \subset \mathbb{R}^{3} \mid \Phi^{0}(x)=0\right\}
$$

as the level set of a $C^{2}$-function

$$
\Phi^{0}: \Omega_{0} \subset \mathbb{R}^{3} \rightarrow \mathbb{R} \text { with }\left|\nabla \Phi^{0}\right| \equiv 1 \text { on } \sigma
$$

defined on an open set $\Omega_{0} \subset \mathbb{R}^{3}$.

Equation (1.3) is Cattaneo's law for heat conduction and turns, as the so-called relaxation parameter $\tau \rightarrow 0$, into Fourier's law

$$
q+\kappa \nabla \theta=0
$$

The system (1.1), (1.2), (1.7) is the hyperbolic-parabolic system of classical thermoelasticity; cp. [6]. The system to be studied here, (1.1)-(1.3), is purely hyperbolic; cp. [2, 8, 9].

The propagation of singularities in the Cauchy problems for the hyperbolic-parabolic coupled system (1.1), (1.2), (1.7) of classical thermoelasticity has been studied in [3, 10, 13, 14 and the references therein. It is observed that the propagation of singularities in this coupled system (even more general nonlinear coupled systems) is dominated by the hyperbolic operators. One of the main tools used in these works is microlocal analysis, which requires a certain regularity of solutions in general. Thus, the above-mentioned works only deal with some weak singularities; in particular, for some important waves, discontinuous solutions have not been investigated.

As pointed out above, formally, when $\tau \rightarrow 0$, the hyperbolic model (1.1), (1.2), (1.3) turns into the hyperbolic-parabolic system. We shall be particularly interested in the behavior of the propagating jumps as $\tau \rightarrow 0$, which should give us some information on the propagation of strong singularities for the hyperbolic-parabolic coupled system. This problem was first studied by authors in [1] for the one-dimensional case. We shall study the multi-dimensional problem in this work. 
By decomposing $(u, q)$ into its solenoidal part $\left(u^{s}, q^{s}\right)\left(\operatorname{div} u^{s}=\operatorname{div} q^{s}=0\right)$ and its potential part $\left(u^{p}, q^{p}\right)\left(\operatorname{curl} u^{p}=\operatorname{curl} q^{p}=0\right)$, the representations and expansions for $\tau \rightarrow 0$ and $t \rightarrow \infty$ of the jumps on the evolving characteristic surfaces are given for the linear problem (Theorem 3.3) and a nonlinear problem (Theorem 4.1). In particular, similar to [11, we shall obtain that the jump of the temperature goes to zero (showing a smoothing effect in the hyperbolic-parabolic coupled systems) while the jumps of the heat flux and the gradient of the potential part of the elastic wave are propagated along the characteristic curves of the elastic fields when the relaxation parameter $\tau$ vanishes.

Moreover, we shall find a very interesting phenomenon: whether the jumps of solutions on the hyperbolic characteristic surfaces decay exponentially when $t \rightarrow+\infty$ depends on which one is dominated between the quantity $\frac{\beta \delta}{2 \kappa \gamma \sqrt{2 \mu+\gamma}}$ and the mean curvature of the initial surface of discontinuity, which is invisible in the one-dimensional work [11. Under certain assumptions, these jumps decay exponentially when time goes to infinity, more rapidly for a smaller heat conductive coefficient, which is similar to the phenomenon observed by Hoff in [4] for discontinuous solutions to the compressible Navier-Stokes equations.

We remark that our considerations could be extended to variable coefficients and the case that nonlinearities $f, g$ depend on $u$ as well. The case that $f, g$ depend on other quantities like $\nabla \theta$ and $\nabla u$ would require further analysis. The two-dimensional case can be studied analogously.

The paper is organized as follows: In Section 2 we give the general setting and a decomposition. The linearized problem $(f=0, g=0)$ is discussed in Section 3, and Section 4 presents the results on a semilinear problem.

2. Setting and decomposition. Consider the following Cauchy problem:

$$
\begin{aligned}
u_{t t}-\mu \Delta u-(\mu+\lambda) \nabla \operatorname{div} u+\beta \nabla \theta & =f\left(u_{t}, \theta\right), \\
\theta_{t}+\gamma \operatorname{div} q+\delta \operatorname{div} u_{t} & =g\left(u_{t}, \theta\right), \\
\tau q_{t}+q+\kappa \nabla \theta & =0
\end{aligned}
$$

on $(0, \infty) \times \mathbb{R}^{3}$,

$$
u(t=0)=u^{0}, u_{t}(t=0)=u^{1}, \theta(t=0)=\theta^{0}, q(t=0)=q^{0}
$$

with data $\left(\nabla u^{0}, u^{1}, \theta^{0}, q^{0}\right)$ being smooth away from and possibly having jumps on a given smooth surface

$$
\sigma=\left\{x \in \Omega_{0} \subset \mathbb{R}^{3} \mid \Phi^{0}(x)=0\right\}
$$

described by a $C^{2}$-function $\Phi^{0}: \Omega_{0} \subset \mathbb{R}^{3} \rightarrow \mathbb{R}$, with $\Omega_{0}$ being an open set and satisfying

$$
\left|\nabla \Phi^{0}(x)\right|=1 \quad \text { on } \quad \sigma .
$$

Assumption (2.5) is important for the following techniques used, but is made without loss of generality, since for a surface $\sigma$ one can choose the distance function

$$
\Phi^{0}(x):=\operatorname{dist}(x, \sigma)
$$

which satisfies (2.5); cp. [5. pp. 354-355]. 
Simple examples are of course spheres of radius $r>0$ with $\Phi^{0}(x)=\frac{1}{2 r}\left(|x|^{2}-r^{2}\right)$ or ellipsoids.

Using the decomposition of vector fields according to the orthogonal decomposition

$$
\left(L^{2}\left(\mathbb{R}^{3}\right)\right)^{3}=\overline{\nabla W^{1,2}\left(\mathbb{R}^{3}\right)} \oplus D_{0}\left(\mathbb{R}^{3}\right),
$$

where $W^{1,2}\left(\mathbb{R}^{3}\right)$ denotes the usual Sobolev space, and $D_{0}\left(\mathbb{R}^{3}\right)$ denotes the set of vector fields having divergence zero, we have

$$
u=u^{p}+u^{s}, q=q^{p}+q^{s}
$$

and (2.1) -2.3$)$ are decomposed into

$$
\begin{gathered}
u_{t t}^{s}-\mu \Delta u^{s}=f^{s}\left(u_{t}, \theta\right), \\
u^{s}(t=0)=u^{0, s}, \quad u_{t}^{s}(t=0)=u^{1, s}
\end{gathered}
$$

and

$$
\begin{aligned}
\tau q_{t}^{s}+q^{s} & =0 \\
q^{s}(t=0) & =q^{0, s}
\end{aligned}
$$

and

$$
\begin{aligned}
u_{t t}^{p}-\alpha^{2} \Delta u^{p}+\beta \nabla \theta & =f^{p}\left(u_{t}, \theta\right), \\
\theta_{t}+\gamma \operatorname{div} q^{p}+\delta \operatorname{div} u_{t}^{p} & =g\left(u_{t}, \theta\right), \\
\tau q_{t}^{p}+q^{p}+\kappa \nabla \theta & =0, \\
u^{p}(t=0)=u^{0, p}, u_{t}^{p}(t=0)=u^{1, p}, \theta(t=0) & =\theta^{0}, q^{p}(t=0)=q^{0, p},
\end{aligned}
$$

where $\alpha:=\sqrt{2 \mu+\lambda}$.

Since (2.9), (2.10) is explicitly solvable, and the propagation of singularities for pure wave equations such as (2.7), (2.8) is well understood, cp. 1, 17, 12, we concentrate on the fully coupled system (2.11)-(2.14). Let

$$
U=\left(U_{1}, U_{2}, U_{3}, U_{4}\right)^{\prime}:=\left(\operatorname{div} u^{p}, u_{t}^{p}, \theta, q^{p}\right)^{\prime}
$$

with the prime denoting the transpose of a vector. Then (2.11)-(2.14) turns into the following system for $U$ :

$$
\begin{aligned}
\partial_{t} U+\sum_{j=1}^{3} A_{j} \partial_{j} U+A_{0} U & =F, \\
U(t=0) & =U^{0},
\end{aligned}
$$

where $\partial_{t}=\frac{\partial}{\partial t}, \partial_{j}=\frac{\partial}{\partial x_{j}}, j=1,2,3$,

$$
\sum_{j \equiv 1}^{3} A_{j} \partial_{j} \equiv\left(\begin{array}{cccc}
0 & -\operatorname{div} & 0 & 0 \\
-\alpha^{2} \nabla & 0 & \beta \nabla & 0 \\
0 & \delta \operatorname{div} & 0 & \gamma \operatorname{div} \\
0 & 0 & \frac{\kappa}{\tau} \nabla & 0
\end{array}\right)
$$

giving $A_{j}$ implicitly, and

$$
A_{0}:=\left(\begin{array}{cc}
0_{5 \times 5} & 0_{5 \times 3} \\
0_{3 \times 5} & \frac{1}{\tau} I d_{\mathbb{R}^{3}}
\end{array}\right), \quad F:=\left(0, f^{p}, g, 0\right)^{\prime} .
$$


3. The linearized problem. We now assume $F=0$; i.e., we consider the linearized version of (2.15), (2.16). For $\xi \in \mathbb{R}^{3} \backslash\{0\}$ we have that the characteristic polynomial

$$
\operatorname{det}\left(\lambda I d_{\mathbb{R}^{8}}-\sum_{j=1}^{3} \xi_{j} A_{j}\right)=\lambda^{4}\left(\lambda^{4}-\lambda^{2}\left(\alpha^{2}+\beta \delta+\frac{\kappa \gamma}{\tau}\right)|\xi|^{2}+\frac{\kappa \gamma \alpha^{2}}{\tau}|\xi|^{4}\right)
$$

has real roots $\lambda_{k}, 1 \leq k \leq 8$, satisfying

$$
\begin{gathered}
\lambda_{k}=0, \quad 1 \leq k \leq 4, \\
\lambda_{k}^{2}=\frac{1}{2}\left(\alpha^{2}+\beta \delta+\frac{\kappa \gamma}{\tau} \pm \sqrt{\left.\left(\alpha^{2}+\beta \delta+\frac{\kappa \gamma}{\tau}\right)^{2}-\frac{4 \kappa \gamma \alpha^{2}}{\tau}\right)}|\xi|^{2} \equiv \mu_{k}^{2}|\xi|^{2}, \quad 5 \leq k \leq 8 .\right.
\end{gathered}
$$

The last factor in (3.1) is similar to the characteristic polynomial in one space dimension, cp. [1], and we know

$$
\lambda_{k} \neq \lambda_{j} \quad \text { for } \quad 5 \leq k \neq j \leq 8 .
$$

The characteristic surfaces $\Sigma_{k}=\left\{(t, x) \mid \Phi_{k}(t, x)=0\right\}, 1 \leq k \leq 8$, evolving from the initial surface $\sigma=\left\{(0, x) \mid \Phi^{0}(x)=0\right\}$ are determined by

$$
\begin{aligned}
\partial_{t} \Phi_{k}+\mu_{k}\left|\nabla \Phi_{k}\right| & =0, \\
\Phi_{k}(0, \cdot) & =\Phi^{0},
\end{aligned}
$$

which is solved by

$$
\begin{aligned}
& \Phi_{k}(t, x)=\Phi^{0}(x), \quad 1 \leq k \leq 4 \\
& \Phi_{k}(t, x)=-\mu_{k} t+\Phi^{0}(x), \quad 5 \leq k \leq 8
\end{aligned}
$$

where we have used the normalization (2.5), $\left|\nabla \Phi^{0}\right|=1$. Hence

$$
\Sigma_{k}=\left\{(t, x) \mid-\mu_{k} t+\Phi^{0}(x)=0\right\} .
$$

Under the assumption (2.5) we have that the eigenvalues of

$$
B:=\sum_{j=1}^{3} \partial_{j} \Phi^{0} A_{j}
$$

are

$$
\begin{gathered}
\lambda_{k}=0, \quad 1 \leq k \leq 4 \\
\lambda_{k}=\mu_{k}= \pm\left\{\frac{1}{2}\left(\alpha^{2}+\beta \delta+\frac{\kappa \gamma}{\tau}\right) \pm \frac{1}{2} \sqrt{\left(\alpha^{2}+\beta \delta+\frac{\kappa \gamma}{\tau}\right)^{2}-\frac{4 \kappa \gamma \alpha^{2}}{\tau}}\right\}^{\frac{1}{2}}, \quad 5 \leq k \leq 8
\end{gathered}
$$

taking

$$
\mu_{8}<\mu_{6}<0<\mu_{7}<\mu_{5} .
$$

Let $\nabla_{\tau_{1}} \Phi^{0}$ and $\nabla_{\tau_{2}} \Phi^{0}$ denote two orthogonal vectors on the tangential plane to the initial surface $\sigma$. Then the associated right and left eigenvectors of $B$ are the column 
vectors $r_{k}$ and the row vectors $l_{k}$,

$$
\begin{aligned}
& r_{1}=c_{1}\left(0,\left(\nabla_{\tau_{1}} \Phi^{0}\right)^{\prime}, 0_{1 \times 4}\right)^{\prime}, \quad r_{2}=c_{2}\left(0,\left(\nabla_{\tau_{2}} \Phi^{0}\right)^{\prime}, 0_{1 \times 4}\right)^{\prime}, \\
& r_{3}=c_{3}\left(0_{1 \times 5},\left(\nabla_{\tau_{1}} \Phi^{0}\right)^{\prime}\right)^{\prime}, \quad r_{4}=c_{4}\left(0_{1 \times 5},\left(\nabla_{\tau_{2}} \Phi^{0}\right)^{\prime}\right)^{\prime}, \\
& r_{k}=\left(\frac{\beta}{\alpha^{2}-\lambda_{k}^{2}},-\frac{\beta \lambda_{k}}{\alpha^{2}-\lambda_{k}^{2}}\left(\nabla \Phi^{0}\right)^{\prime}, 1, \frac{\kappa}{\tau \lambda_{k}}\left(\nabla \Phi^{0}\right)^{\prime}\right)^{\prime}, \quad 5 \leq k \leq 8, \\
& l_{1}=\left(0,\left(\nabla_{\tau_{1}} \Phi^{0}\right)^{\prime}, 0_{1 \times 4}\right), \quad l_{2}=\left(0,\left(\nabla_{\tau_{2}} \Phi^{0}\right)^{\prime}, 0_{1 \times 4}\right), \\
& l_{3}=\left(0_{1 \times 5},\left(\nabla_{\tau_{1}} \Phi^{0}\right)^{\prime}\right), \quad l_{4}=\left(0_{1 \times 5},\left(\nabla_{\tau_{2}} \Phi^{0}\right)^{\prime}\right), \\
& l_{k}=c_{k}\left(\frac{\alpha^{2} \delta}{\alpha^{2}-\lambda_{k}^{2}},-\frac{\delta \lambda_{k}}{\alpha^{2}-\lambda_{k}^{2}}\left(\nabla \Phi^{0}\right)^{\prime}, 1, \frac{\gamma}{\lambda_{k}}\left(\nabla \Phi^{0}\right)^{\prime}\right), \quad 5 \leq k \leq 8,
\end{aligned}
$$

where $c_{k}, 1 \leq k \leq 8$, is chosen such that the normalization condition

$$
l_{j} r_{k}= \begin{cases}1, & j=k \\ 0, & j \neq k\end{cases}
$$

holds. For example, we can set

$$
c_{1}=c_{3}=\frac{1}{\left|\nabla \tau_{1} \Phi^{0}\right|^{2}}, \quad c_{2}=c_{4}=\frac{1}{\left|\nabla \tau_{2} \Phi^{0}\right|^{2}}
$$

and

$$
1=c_{k}\left(\frac{\alpha^{2} \beta \delta}{\left(\alpha^{2}-\lambda_{k}^{2}\right)^{2}}+\frac{\beta \delta \lambda_{k}^{2}}{\left(\alpha^{2}-\lambda_{k}^{2}\right)^{2}}+1+\frac{\kappa \gamma}{\tau \lambda_{k}^{2}}\right), \quad 5 \leq k \leq 8 .
$$

If $k=5,6$ we have the expansion, as $\tau \rightarrow 0$,

$$
\begin{aligned}
\mu_{k}^{2}=\lambda_{k}^{2} & =\frac{1}{2}\left(\alpha^{2}+\beta \delta+\frac{\kappa \gamma}{\tau}+\sqrt{\left(\alpha^{2}+\beta \delta+\frac{\kappa \gamma}{\tau}\right)^{2}-\frac{4 \kappa \gamma \alpha^{2}}{\tau}}\right) \\
& =\frac{\kappa \gamma}{\tau}+\beta \delta+\frac{\alpha^{2} \beta \delta}{\kappa \gamma} \tau+\mathcal{O}\left(\tau^{2}\right)
\end{aligned}
$$

(cp. [11) and for $k=7,8$,

$$
\mu_{k}^{2}=\lambda_{k}^{2}=\frac{1}{2}\left(\alpha^{2}+\beta \delta+\frac{\kappa \gamma}{\tau}-\sqrt{\left(\alpha^{2}+\beta \delta+\frac{\kappa \gamma}{\tau}\right)^{2}-\frac{4 \kappa \gamma \alpha^{2}}{\tau}}\right)=\alpha^{2}-\frac{\alpha^{2} \beta \delta}{\kappa \gamma} \tau+\mathcal{O}\left(\tau^{2}\right) .
$$

Thus for $k=5,6$, formula (3.22) reads

$$
\begin{aligned}
1 & =c_{k}\left(1+\frac{\kappa \gamma}{\kappa \gamma+\beta \delta \tau+\frac{\alpha^{2} \beta \delta}{\kappa \gamma} \tau^{2}+\mathcal{O}\left(\tau^{3}\right)}+\beta \delta \frac{\alpha^{2}+\beta \delta+\frac{\kappa \gamma}{\tau}+\frac{\alpha^{2} \beta \delta}{\kappa \gamma} \tau+\mathcal{O}\left(\tau^{2}\right)}{\left(\frac{\kappa \gamma}{\tau}+\beta \delta-\alpha^{2}+\frac{\alpha^{2} \beta \delta}{\kappa \gamma} \tau+\mathcal{O}\left(\tau^{2}\right)\right)^{2}}\right) \\
& =c_{k}\left(2+\mathcal{O}\left(\tau^{2}\right)\right),
\end{aligned}
$$

which implies

$$
c_{k}=\frac{1}{2}+\mathcal{O}\left(\tau^{2}\right), \quad k=5,6
$$


while for $k=7,8$ we get

$$
\begin{aligned}
1 & =c_{k}\left(1+\frac{\kappa \gamma}{\alpha^{2} \tau-\frac{\alpha^{2} \beta \delta}{\kappa \gamma} \tau^{2}+\mathcal{O}\left(\tau^{3}\right)}+\beta \delta \frac{2 \alpha^{2}-\frac{\alpha^{2} \beta \delta}{\kappa \gamma} \tau+\mathcal{O}\left(\tau^{2}\right)}{\left(\frac{\alpha^{2} \beta \delta}{\kappa \gamma} \tau+\mathcal{O}\left(\tau^{2}\right)\right)^{2}}\right) \\
& =c_{k} \frac{2 \kappa^{2} \gamma^{2}}{\alpha^{2} \beta \delta} \tau^{-2}(1+\mathcal{O}(\tau)),
\end{aligned}
$$

which gives

$$
c_{k}=\frac{\alpha^{2} \beta \delta}{2 \kappa^{2} \gamma^{2}} \tau^{2}+\mathcal{O}\left(\tau^{3}\right), \quad k=7,8
$$

Let the matrices $L$ and $R$ be given by

$$
L:=\left(\begin{array}{c}
l_{1} \\
\vdots \\
l_{8}
\end{array}\right), \quad R:=\left(r_{1}, \ldots, r_{8}\right) .
$$

Then $V:=L U$, with $U$ satisfying (2.15), (2.16), satisfies

$$
\begin{aligned}
& \partial_{t} V+\sum_{j=1}^{3}\left(L A_{j} R\right) \partial_{j} V+\widetilde{A}_{0} V=\widetilde{F}, \\
& V(t=0)=V^{0}:=L U^{0},
\end{aligned}
$$

where

$$
\begin{aligned}
\widetilde{A}_{0} & :=L A_{0} R+\sum_{j=1}^{3} L A_{j} \partial_{j} R, \\
\widetilde{F} & :=L F \quad(=0 \text { in this section }) .
\end{aligned}
$$

Then we have as the first result, where $[H]_{\Sigma_{k}}$ denotes the jump of $H$ along $\Sigma_{k}$ :

Lemma 3.1. Let $V_{I}:=\left(V_{1}, V_{2}, V_{3}, V_{4}\right)$. Then,

$$
\begin{aligned}
& {\left[V_{I}\right]_{\Sigma_{k}}=0, \quad 5 \leq k \leq 8,} \\
& {\left[V_{j}\right]_{\Sigma_{k}}=0, \quad 5 \leq j \leq 8, \quad k=1, \ldots, 8, \quad j \neq k ;}
\end{aligned}
$$

i.e., the $V_{I}$ are continuous at $\bigcup_{k=5}^{8} \Sigma_{k}$, and $V_{j}, j=5, \ldots, 8$, does not have any jump on $\Sigma_{k}, k=1, \ldots, 8$ for $k \neq j$.

Proof. Let $V$ be a bounded piecewise smooth solution to (3.29), (3.30) with possible discontinuities only on $\Sigma_{k}, k=0,5,6,7,8$, where $\Sigma_{0}:=\left\{(t, x) \in \Omega \mid \Phi^{0}(x)=0\right\}$.

For a fixed $k \in\{5,6,7,8\}$, let $\Omega$ be a bounded domain in $\mathbb{R}^{+} \times \mathbb{R}^{3}$ containing only a part of $\Sigma_{k}$ while $\Sigma_{j} \cap \Omega=\emptyset$ for $j \neq k$. Let

$$
\Omega^{ \pm}=\left\{(t, x) \in \Omega \mid \pm \Phi_{k}(t, x)>0\right\} .
$$

The equation (3.29) holds in $\Omega$ in the distribution sense; that is, we have for any function $\varphi \in\left(C_{0}^{\infty}(\Omega)\right)^{8}$,

$$
\int_{\Omega}\left(V \cdot \partial_{t} \varphi+\sum_{j=1}^{3} \partial_{j}\left(\varphi^{*} L A_{j} R\right) V\right) d x d t=-\int_{\Omega}\left(\widetilde{F}-\widetilde{A}_{0} V\right) \cdot \varphi d x d t
$$


Using the Gauss integral theorem for the left-hand side on $\Omega^{ \pm}$, respectively, and observing that (3.29) holds in the classical sense in $\Omega^{ \pm}$, it follows that

$$
\int_{\Omega \cap \Sigma_{k}}\left([V]_{\Sigma_{k}} \vec{n}_{0}+\sum_{j=1}^{3} L A_{j} R[V]_{\Sigma_{k}} \vec{n}_{j}\right) \cdot \varphi=0,
$$

where $\vec{n}=\left(\vec{n}_{0}, \vec{n}_{1}, \vec{n}_{2}, \vec{n}_{3}\right)^{\prime}$ denotes the unit normal vector on $\Sigma_{k}$. Since $\Sigma_{k}$ $=\left\{(t, x) \mid \Phi_{k}(t, x)=0\right\}$ we have

$$
\vec{n} \|\left(\lambda_{k},-\left(\nabla \Phi^{0}\right)^{\prime}\right) .
$$

This implies, since $\varphi$ is arbitrary in $\left(C_{0}^{\infty}(\Omega)\right)^{8}$,

$$
\left(\lambda_{k} I d_{\mathbb{R}^{8}}-\sum_{j=1}^{3} L A_{j} R \partial_{j} \Phi^{0}\right)[V]_{\Sigma_{k}}=0
$$

or

$$
\left(L\left(\lambda_{k} I d_{\mathbb{R}^{8}}-\sum_{j=1}^{3}\left(\partial_{j} \Phi^{0}\right) A_{j}\right) R\right)[V]_{\Sigma_{k}}=0 ;
$$

hence, by the definitions of $L$ and $R$,

$$
\left(\lambda_{k} I d_{\mathbb{R}^{8}}-\widetilde{\Lambda}\right)[V]_{\Sigma_{k}}=0,
$$

where

$$
\widetilde{\Lambda}:=\operatorname{diag}\left(\lambda_{1}, \ldots, \lambda_{8}\right) .
$$

This implies

$$
\left[V_{j}\right]_{\Sigma_{k}}=0
$$

for $k=5, \ldots, 8$ and $j=1, \ldots, 4$, or $k=1, \ldots, 8$ and $j=5, \ldots, 8$ and $j \neq k$.

From Lemma 3.1, we know that it remains to study the behavior of $V_{k}$ on $\Sigma_{k}$. First, we have

Lemma 3.2. For $1 \leq k \leq 4$ we have

$$
\left[V_{k}\right]_{\Sigma_{0}}=0
$$

Proof. By definition we have

$$
V_{k}=l_{k} U, \quad k=1, \ldots, 4
$$

or

$$
V_{1}=\partial_{t}\left(u^{p} \cdot \nabla_{\tau_{1}} \Phi^{0}\right), \quad V_{2}=\partial_{t}\left(u^{p} \cdot \nabla_{\tau_{2}} \Phi^{0}\right), \quad V_{3}=q^{p} \cdot \nabla_{\tau_{1}} \Phi^{0}, \quad V_{4}=q^{p} \cdot \nabla_{\tau_{2}} \Phi^{0} .
$$

For $\omega \in\left\{u^{p}, q^{p}\right\}$ we have curl $\omega=0$, classically away from $\Sigma_{0}$, and always in the distributional sense; that is, for a bounded domain $G \subset \mathbb{R}_{t}^{+} \times \mathbb{R}^{3}$ with $\Sigma_{0} \cap G \neq \phi$ and $\varphi \in C_{0}^{\infty}(G)$ we have

implying

$$
\int_{G} \omega \operatorname{curl} \varphi=0
$$

$$
\int_{\Sigma_{0}}\left([\omega]_{\Sigma_{0}} \times \vec{\nu}\right) \varphi=0
$$


where $\vec{\nu}$ denotes the normal on $\Sigma_{0}$ and is parallel to $\nabla \Phi^{0}$. Hence we get, since $\varphi$ is arbitrary,

$$
[w]_{\Sigma_{0}} \times \vec{\nu}=0
$$

which gives

$$
[w]_{\Sigma_{0}}=0 \quad \text { or } \quad[w]_{\Sigma_{0}} \| \vec{\nu}
$$

the latter implying

$$
\left[w \cdot \nabla_{\tau_{m}} \Phi^{0}\right]_{\Sigma_{0}}=0, \quad m=1,2 .
$$

The assertion of the lemma now follows from (3.35)-(3.37).

Next, let us derive the evolutional equations of $\left[V_{k}\right]_{\Sigma_{k}}$ for $5 \leq k \leq 8$.

Denote by $D_{m k}$ the $m$-th row and $k$-th column element of an $(8 \times 8)$-matrix $D$. Obviously, the $k$-th equation given in (3.29) can be explicitly written as

$$
\partial_{t} V_{k}+\sum_{m=1}^{8} \sum_{j=1}^{3}\left(L A_{j} R\right)_{k m} \partial_{j} V_{m}=-\sum_{m=1}^{8}\left(\widetilde{A}_{0}\right)_{k m} V_{m}+\widetilde{F_{k}}
$$

for $5 \leq k \leq 8$.

From the fact that

$$
\left(\lambda_{k} I d_{\mathbb{R}^{8}}-\sum_{j=1}^{3} L \partial_{j} \Phi^{0} A_{j} R\right)_{k k}=\left(\lambda_{k} I d_{\mathbb{R}^{8}}-\widetilde{\Lambda}\right)_{k k}=0
$$

with $\widetilde{\Lambda}=\operatorname{diag}\left(\lambda_{1}, \ldots, \lambda_{8}\right)$, we know that the operator $\partial_{t}+\sum_{j=1}^{3}\left(L A_{j} R\right)_{k k} \partial_{j}$ is tangential to $\Sigma_{k}=\left\{\lambda_{k} t+\Phi^{0}(x)=0\right\}$. Similarly, since all the entries in line $k$ of $\lambda_{k} I d_{\mathbb{R}^{8}}-\sum_{j=1}^{3} L \partial_{j} \Phi^{0} A_{j} R=\lambda_{k} I d_{\mathbb{R}^{8}}-\widetilde{\Lambda}$ vanish, we deduce that for $m \neq k$, $\left(0,\left(L A_{1} R\right)_{k m},\left(L A_{2} R\right)_{k m},\left(L A_{3} R\right)_{k m}\right)$ is orthogonal to the normal direction $\left(-\lambda_{k}\right.$, $\left.\left(\nabla \Phi^{0}\right)^{\prime}\right)$ of $\Sigma_{k}$, which implies that $\sum_{j=1}^{3}\left(L A_{j} R\right)_{k m} \partial_{j}$ is tangential to $\Sigma_{k}$ when $m \neq k$.

Therefore, by using Lemmas 3.1 and 3.2 in (3.38), we obtain that for each $5 \leq k \leq 8$, $\left[V_{k}\right]_{\Sigma_{k}}$ satisfies the following transport equation:

$$
\left(\partial_{t}+\sum_{j=1}^{3}\left(L A_{j} R\right)_{k k} \partial_{x_{j}}+\left(\widetilde{A}_{0}\right)_{k k}\right)\left[V_{k}\right]_{\Sigma_{k}}=\left[\tilde{F}_{k}\right]_{\Sigma_{k}}
$$

with initial conditions

$$
\left[V_{k}\right]_{\Sigma_{k}}(t=0)=\left[V_{k}^{0}\right]_{\sigma} .
$$

In order to determine the behavior of $\left[V_{k}\right]_{\Sigma_{k}}$ from (3.40), (3.41), it is essential to study $\left(\widetilde{A}_{0}\right)_{k k}$, where, by (3.31), $\widetilde{A}_{0}$ is given by

$$
\widetilde{A}_{0}=L A_{0} R+\sum_{j=1}^{3} L A_{j} \partial_{j} R
$$

Denoting the row $l_{k}$ and the column $r_{k}$ by $l_{k}=\left(l_{k 1}, \ldots, l_{k 8}\right)$, resp. $r_{k}=\left(r_{1 k}, \ldots, r_{8 k}\right)^{\prime}$, we have, using (2.18), for $k=5, \ldots, 8$,

$$
\left(L A_{0} R\right)_{k k}=\frac{1}{\tau} \sum_{j=6}^{8} l_{k j} r_{j k}
$$


hence, using (3.16), (3.19),

$$
\left(L A_{0} R\right)_{k k}=\frac{c_{k} \kappa \gamma}{\tau^{2} \lambda_{k}^{2}}
$$

which implies for $k=5,6$, using (3.23), (3.26),

$$
\left(L A_{0} R\right)_{k k}=\left(\frac{1}{2}+\mathcal{O}\left(\tau^{2}\right)\right) \frac{\kappa \gamma}{\tau^{2}\left(\frac{k \gamma}{\tau}+\beta \delta+\frac{\alpha^{2} \beta \delta}{\kappa \gamma} \tau+\mathcal{O}\left(\tau^{2}\right)\right)}=\frac{1}{2 \tau}-\frac{\beta \delta}{2 \kappa \gamma}+\mathcal{O}(\tau)
$$

and for $k=7,8$, using (3.24), (3.28),

$$
\left(L A_{0} R\right)_{k k}=\left(\frac{\alpha^{2} \beta \delta}{2 \kappa^{2} \gamma^{2}} \tau^{2}+\mathcal{O}\left(\tau^{3}\right)\right) \frac{\kappa \gamma}{\tau^{2}\left(\alpha^{2}-\frac{\alpha^{2} \beta \delta}{\kappa \gamma} \tau+\mathcal{O}\left(\tau^{2}\right)\right)}=\frac{\beta \delta}{2 \kappa \gamma}+\mathcal{O}(\tau) .
$$

Now we compute $\left(\sum_{j=1}^{3} L A_{j} \partial_{j} R\right)_{k k}$. Let $\vec{e}_{j}$ be the $j$-th standard unit vector having only zero components but a 1 in the $j$-th component. Then

$$
A_{1}=\left(\begin{array}{cccc}
0 & -\vec{e}_{1}^{\prime} & 0 & 0 \\
-\alpha^{2} \vec{e}_{1} & 0 & \beta \vec{e}_{1} & 0 \\
0 & \delta \vec{e}_{1}^{\prime} & 0 & \gamma \vec{e}_{1}^{\prime} \\
0 & 0 & \frac{\kappa}{\tau} \vec{e}_{1} & 0
\end{array}\right)
$$

and $A_{2}, A_{3}$ arise from $A_{1}$ by replacing $\vec{e}_{1}$ by $\vec{e}_{2}$ and $\vec{e}_{3}$, respectively.

Denote by $L A_{j} \partial_{j} R=\left(a_{i k}^{j}\right)_{8 \times 8}$ for any $j=1,2,3$. Then, by a direct computation, we deduce that for $k=5, \ldots, 8$,

$$
\begin{aligned}
& a_{k k}^{1}=-l_{k 1} \partial_{1} r_{2 k}+l_{k 2}\left(\beta \partial_{1} r_{5 k}-\alpha^{2} \partial_{1} r_{1 k}\right)+l_{k 5}\left(\delta \partial_{1} r_{2 k}+\gamma \partial_{1} r_{6 k}\right)+l_{k 6} \frac{\kappa}{\tau} \partial_{1} r_{5 k}, \\
& a_{k k}^{2}=-l_{k 1} \partial_{2} r_{3 k}+l_{k 3}\left(\beta \partial_{2} r_{5 k}-\alpha^{2} \partial_{2} r_{1 k}\right)+l_{k 5}\left(\delta \partial_{2} r_{3 k}+\gamma \partial_{2} r_{7 k}\right)+l_{k 7} \frac{\kappa}{\tau} \partial_{2} r_{5 k}, \\
& a_{k k}^{3}=-l_{k 1} \partial_{3} r_{4 k}+l_{k 4}\left(\beta \partial_{3} r_{5 k}-\alpha^{2} \partial_{3} r_{1 k}\right)+l_{k 5}\left(\delta \partial_{3} r_{4 k}+\gamma \partial_{3} r_{8 k}\right)+l_{k 8} \frac{\kappa}{\tau} \partial_{3} r_{5 k},
\end{aligned}
$$

which gives

$$
\begin{aligned}
\left(\sum_{j=1}^{3} L A_{j} \partial_{j} R\right)_{k k}= & -l_{k 1}\left(\partial_{1} r_{2 k}+\partial_{2} r_{3 k}+\partial_{3} r_{4 k}\right) \\
& +l_{k 5}\left(\delta\left(\partial_{1} r_{2 k}+\partial_{2} r_{3 k}+\partial_{3} r_{4 k}\right)+\gamma\left(\partial_{1} r_{6 k}+\partial_{2} r_{7 k}+\partial_{3} r_{8 k}\right)\right) .
\end{aligned}
$$

Using (3.16) again we conclude

$$
\left(\sum_{j=1}^{3} L A_{j} \partial_{j} R\right)_{k k}=\left(\left(-\delta l_{k 5}+l_{k 1}\right) \frac{\beta \lambda_{k}}{\alpha^{2}-\lambda_{k}^{2}}+l_{k 5} \frac{\kappa \gamma}{\tau \lambda_{k}}\right) \Delta \Phi^{0},
$$

where $\Delta$ denotes the Laplace operator in $\mathbb{R}^{3}$. Since

$$
\delta l_{k 5}-l_{k 1}=c_{k}\left(\delta-\frac{\alpha^{2} \delta}{\alpha^{2}-\lambda_{k}^{2}}\right)=-c_{k} \frac{\delta \lambda_{k}^{2}}{\alpha^{2}-\lambda_{k}^{2}}
$$

we get from (3.45),

$$
\left(\sum_{j=1}^{3} L A_{j} \partial_{j} R\right)_{k k}=c_{k}\left(\frac{\beta \delta \lambda_{k}^{3}}{\left(\alpha^{2}-\lambda_{k}^{2}\right)^{2}}+\frac{\kappa \gamma}{\tau \lambda_{k}}\right) \Delta \Phi^{0} .
$$


Using the expansions (3.23), (3.26), we obtain for $k=5,6$,

$$
\begin{aligned}
& c_{k}\left(\frac{\beta \delta \lambda_{k}^{3}}{\left(\alpha^{2}-\lambda_{k}^{2}\right)^{2}}+\frac{\kappa \gamma}{\tau \lambda_{k}}\right)=\left(\frac{1}{2}+\mathcal{O}\left(\tau^{2}\right)\right) \frac{1}{\lambda_{k}}\left(\frac{\kappa \gamma}{\tau}+\beta \delta+\mathcal{O}(\tau)\right), \\
& \frac{1}{\lambda_{k}}= \pm \sqrt{\frac{\tau}{\kappa \gamma}}(1+\mathcal{O}(\tau)),
\end{aligned}
$$

implying

$$
\left(\sum_{j=1}^{3} L A_{j} \partial_{j} R\right)_{k k}= \pm \frac{1}{2} \sqrt{\frac{\kappa \gamma}{\tau}}(1+\mathcal{O}(\tau)) \Delta \Phi^{0}, \quad k=5,6 .
$$

Similarly we obtain, using the expansions (3.24), (3.28),

$$
\left(\sum_{j=1}^{3} L A_{j} \partial_{j} R\right)_{k k}= \pm\left(\frac{\alpha}{2}+\mathcal{O}(\tau)\right) \Delta \Phi^{0}, \quad k=7,8 .
$$

Combining (3.42), (3.43), (3.44), (3.47) with (3.48), we obtain

$$
\left(\widetilde{A}_{0}\right)_{k k}= \begin{cases}\frac{1}{2 \tau} \pm \frac{1}{2} \sqrt{\frac{\kappa \gamma}{\tau}} \Delta \Phi^{0}+\mathcal{O}(1), & k=5,6, \\ \frac{\beta \delta}{2 \kappa \gamma} \pm \frac{\alpha}{2} \Delta \Phi^{0}+\mathcal{O}(\tau), & k=7,8 .\end{cases}
$$

From (3.49) we already notice the phenomenon that the mean curvature $H$ of the initial surface $\sigma$,

$$
H=\frac{\Delta \Phi^{0}}{2}
$$

will play an essential role in the behavior of the jumps as $t \rightarrow \infty$ or as $\tau \rightarrow 0$.

For any fixed $x^{0} \in \sigma$, denote by

$$
t \rightarrow\left(t, x_{1}\left(t ; 0, x^{0}\right), x_{2}\left(t ; 0, x^{0}\right), x_{3}\left(t ; 0, x^{0}\right)\right)
$$

the characteristic line of the operator $\partial_{t}+\sum_{j=1}^{3}\left(L A_{j} R\right)_{k k} \partial_{x_{j}}$ passing through $\left(0, x^{0}\right)$; i.e., $x_{j}\left(t ; 0, x^{0}\right)$ satisfies

$$
\left\{\begin{array}{l}
\frac{\partial x_{j}\left(t ; 0, x^{0}\right)}{\partial t}=\left(L A_{j} R\right)_{k k}\left(t, x_{1}\left(t ; 0, x^{0}\right), x_{2}\left(t ; 0, x^{0}\right), x_{3}\left(t ; 0, x^{0}\right)\right), \\
x_{j}\left(0 ; 0, x^{0}\right)=x_{j}^{0}, \quad 1 \leq j \leq 3
\end{array}\right.
$$

and

$$
\left[V_{k}\right]_{\Sigma_{k}(t)}=\left[V_{k}\right]_{\Sigma_{k}}\left(t, x_{1}\left(t ; 0, x^{0}\right), x_{2}\left(t ; 0, x^{0}\right), x_{3}\left(t ; 0, x^{0}\right)\right) .
$$

Then, from (3.40) and (3.49) we conclude:

$$
\begin{aligned}
{\left[V_{k}\right]_{\Sigma_{k}(t)} } & =\left[V_{k}^{0}\right]_{\sigma} e^{-\int_{0}^{t}\left(\widetilde{A}_{0}\right)_{k k}\left(x\left(s ; 0, x^{0}\right)\right) d s} \\
& = \begin{cases}e^{-\frac{t}{2 \tau} \mp \frac{1}{2} \sqrt{\frac{\kappa \gamma}{\tau}} \int_{0}^{t}\left(\Delta \Phi^{0}\left(x\left(s ; 0, x^{0}\right)\right)+O(\sqrt{\tau})\right) d s}\left[V_{k}^{0}\right]_{\sigma}, & k=5,6, \\
e^{-\frac{\beta \delta}{2 \kappa \gamma} t \mp \frac{\alpha}{2} \int_{0}^{t}\left(\Delta \Phi^{0}\left(x\left(s ; 0, x^{0}\right)\right)+\mathcal{O}(\tau)\right) d s}\left[V_{k}^{0}\right]_{\sigma}, & k=7,8 .\end{cases}
\end{aligned}
$$

For $\tau \rightarrow 0$ the dominating term for $k=5,6$ is $e^{-\frac{t}{2 \tau}}$; i.e., we have exponential decay of the jumps of $V_{k}$ on $\Sigma_{k}$ as $\tau \rightarrow 0$ or $t \rightarrow \infty$ for a fixed small $\tau>0$. If $k=7,8$, the dominating term, for $\tau \rightarrow 0$, is $\exp \left(-\int_{0}^{t}\left(\frac{\beta \delta}{2 \kappa \gamma} \pm \frac{\alpha}{2} \Delta \Phi^{0}\left(x\left(s ; 0, x^{0}\right)\right)\right) d s\right)$; whether the jumps of $V_{k}$ on $\Sigma_{k}$ decay exponentially depends on the size of the mean curvature $\left(=\Delta \Phi^{0} / 2\right)$. 
EXAMPLE 3.1. Let $\sigma$ be the sphere of radius $r$ :

$$
\sigma=\left\{x \in \mathbb{R}^{3}|| x \mid=r\right\}=\left\{x\left|\Phi^{0}(x) \equiv r-\right| x \mid=0\right\} .
$$

Then, we have

$$
\Sigma_{k}=\left\{(t, x)\left|\mu_{k} t=r-\right| x \mid\right\}=\left\{(t, x)|| x \mid=r-\mu_{k} t\right\} .
$$

Remember the convention on the signs of $\mu_{k}$ given in (3.13). Spreading as $t \rightarrow \infty$ are $\Sigma_{6}, \Sigma_{8}$, and

$$
\Delta \Phi^{0}\left(x_{0}\right)=\frac{2}{\left|x_{0}\right|}=\frac{2}{r}>0 .
$$

Thus, as $t \rightarrow+\infty,\left[V_{6}\right]_{\Sigma_{6}}$ is decaying exponentially, while $\left[V_{8}\right]_{\Sigma_{8}}$ decays (resp. grows) exponentially if

$$
\frac{\beta \delta}{\alpha \kappa \gamma}>\Delta \Phi^{0}=\frac{2}{r} \quad\left(\frac{\beta \delta}{\alpha \kappa \gamma}<\Delta \Phi^{0}=\frac{2}{r} \text { resp. }\right),
$$

that is, depending on the size of the mean curvature $H=\frac{1}{r}$.

To complete the discussion on the linear problem, we have to compute $U=R V$ from $V$,

$$
U=\left(\operatorname{div} u^{p}, u_{t}^{p}, \theta, q^{p}\right)^{\prime}=R V=\sum_{k=1}^{8} V_{k} r_{k} .
$$

Using (3.14) $-(3.16)$ we conclude

$$
\begin{aligned}
\operatorname{div} u^{p} & =\sum_{k=5}^{8} \frac{\beta}{\alpha^{2}-\lambda_{k}^{2}} V_{k}, \\
u_{t}^{p} & =-\sum_{k=5}^{8} \frac{\beta \lambda_{k}}{\alpha^{2}-\lambda_{k}^{2}} \nabla \Phi^{0} V_{k}+\frac{\nabla_{\tau_{1}} \Phi^{0}}{\left|\nabla_{\tau_{1}} \Phi^{0}\right|^{2}} V_{1}+\frac{\nabla_{\tau_{2}} \Phi^{0}}{\left|\nabla_{\tau_{2}} \Phi^{0}\right|^{2}} V_{2}, \\
\theta & =\sum_{k=5}^{8} V_{k}, \\
q^{p} & =\sum_{k=5}^{8} \frac{\kappa}{\tau \lambda_{k}} \nabla \Phi^{0} V_{k}+\frac{\nabla_{\tau_{1}} \Phi^{0}}{\left|\nabla_{\tau_{1}} \Phi^{0}\right|^{2}} V_{3}+\frac{\nabla_{\tau_{2}} \Phi^{0}}{\left|\nabla_{\tau_{2}} \Phi^{0}\right|^{2}} V_{4} .
\end{aligned}
$$

Observing $\left[V_{j}\right]_{\Sigma_{k}}=0$ for $j=1, \ldots, 4$ and $k=5, \ldots, 8$, cp. Lemmas 3.1 and 3.2, we get from (3.53)-3.56) for $k=5, \ldots, 8$ :

$$
\begin{aligned}
{\left[\operatorname{div} u^{p}\right]_{\Sigma_{k}} } & =\frac{\beta}{\alpha^{2}-\lambda_{k}^{2}}\left[V_{k}\right]_{\Sigma_{k}}, \\
{\left[u_{t}^{p}\right]_{\Sigma_{k}} } & =-\frac{\beta \lambda_{k}}{\alpha^{2}-\lambda_{k}^{2}} \nabla \Phi^{0}\left[V_{k}\right]_{\Sigma_{k}} \\
{[\theta]_{\Sigma_{k}} } & =\left[V_{k}\right]_{\Sigma_{k}} \\
{\left[q^{p}\right]_{\Sigma_{k}} } & =\frac{\kappa}{\tau \lambda_{k}} \nabla \Phi^{0}\left[V_{k}\right]_{\Sigma_{k}} .
\end{aligned}
$$


Using the expansions

$$
\begin{aligned}
\frac{1}{\alpha^{2}-\lambda_{k}^{2}} & = \begin{cases}-\frac{\tau}{\kappa \gamma}+\mathcal{O}\left(\tau^{2}\right), & k=5,6, \\
\frac{\kappa \gamma}{\alpha^{2} \beta \delta} \frac{1}{\tau}+\mathcal{O}(1), & k=7,8,\end{cases} \\
\lambda_{k} & = \begin{cases} \pm \sqrt{\frac{\kappa \gamma}{\tau}}(1+\mathcal{O}(\tau)), & k=5,6, \\
\pm \alpha+\mathcal{O}(\tau), & k=7,8,\end{cases}
\end{aligned}
$$

we conclude from (3.57)-(3.60), using (3.51),

$$
\begin{aligned}
{\left[\operatorname{div} u^{p}\right]_{\Sigma_{k}} } & =\left\{\begin{array}{l}
-\frac{\beta}{\kappa \gamma}\left(\tau+\mathcal{O}\left(\tau^{2}\right)\right) e^{-\frac{t}{2 \tau}(1+\mathcal{O}(\sqrt{\tau}))}\left[V_{k}^{0}\right]_{\sigma}, \quad k=5,6, \\
\frac{\kappa \gamma}{\alpha^{2} \delta}\left(\frac{1}{\tau}+\mathcal{O}(1)\right) e^{-\int_{0}^{t}\left(\frac{\beta \delta}{2 \kappa \gamma} \pm \frac{\alpha}{2} \Delta \Phi^{0}\left(x\left(s ; 0, x^{0}\right)\right)+\mathcal{O}(\tau)\right) d s}\left[V_{k}^{0}\right]_{\sigma}, \quad k=7,8
\end{array}\right. \\
{\left[u_{t}^{p}\right]_{\Sigma_{k}} } & =\left\{\begin{array}{l} 
\pm \frac{\beta}{\sqrt{\kappa \gamma}} \nabla \Phi^{0} \sqrt{\tau}(1+\mathcal{O}(\tau)) e^{-\frac{t}{2 \tau}(1+\mathcal{O}(\sqrt{\tau}))}\left[V_{k}^{0}\right]_{\sigma}, \quad k=5,6 \\
\mp \frac{\kappa \gamma}{\alpha \delta}\left(\frac{1}{\tau}+\mathcal{O}(1)\right) e^{-\int_{0}^{t}\left(\frac{\beta \delta}{2 \kappa \gamma} \pm \frac{\alpha}{2} \Delta \Phi^{0}\left(x\left(s ; 0, x^{0}\right)\right)+\mathcal{O}(\tau)\right) d s}\left[V_{k}^{0}\right]_{\sigma}, \quad k=7,8
\end{array}\right. \\
{[\theta]_{\Sigma_{k}} } & =\left\{\begin{array}{l}
e^{-\frac{t}{2 \tau}(1+\mathcal{O}(\sqrt{\tau}))}\left[V_{k}^{0}\right]_{\sigma}, \quad k=5,6, \\
e^{-\int_{0}^{t}\left(\frac{\beta \delta}{2 \kappa \gamma} \pm \frac{\alpha}{2} \Delta \Phi^{0}\left(x\left(s ; 0, x^{0}\right)\right)+\mathcal{O}(\tau)\right) d s}\left[V_{k}^{0}\right]_{\sigma}, \quad k=7,8,
\end{array}\right. \\
{\left[q^{p}\right]_{\Sigma_{k}} } & =\left\{\begin{array}{l} 
\pm \sqrt{\frac{\kappa}{\gamma}} \nabla \Phi^{0}\left(\frac{1}{\sqrt{\tau}}+\mathcal{O}(\sqrt{\tau})\right) e^{-\frac{t}{2 \tau}(1+\mathcal{O}(\sqrt{\tau}))}\left[V_{k}^{0}\right]_{\sigma}, \quad k=5,6, \\
\pm \frac{\kappa}{\alpha} \nabla \Phi^{0}\left(\frac{1}{\tau}+\mathcal{O}(1)\right) e^{-\int_{0}^{t}\left(\frac{\beta \delta}{2 \kappa \gamma} \pm \frac{\alpha}{2} \Delta \Phi^{0}\left(x\left(s ; 0, x^{0}\right)\right)+\mathcal{O}(\tau)\right) d s}\left[V_{k}^{0}\right]_{\sigma}, \quad k=7,8 .
\end{array}\right.
\end{aligned}
$$

We see from (3.63)-(3.66) that if $\left[V_{k}^{0}\right]_{\sigma} \neq 0, k=5, \ldots, 8$, then the jumps of $\left(\operatorname{div} u^{p}, u_{t}^{p}, \theta, q^{p}\right)$ on $\Sigma_{k}(k=5,6)$ decay exponentially as $\tau \rightarrow 0$, and for fixed small $\tau$, they decay exponentially as well when $t \rightarrow \infty$, while the behavior of those on $\Sigma_{k}$ $(k=7,8)$ depends on the mean curvature $\left(\frac{1}{2} \Delta \Phi^{0}\right)$ of the initial surface $\sigma$, even allowing exponential growth.

Obviously, from the transformation $V=L \cdot\left(\operatorname{div} u^{p}, u_{t}^{p}, \theta, q^{p}\right)^{\prime}$, we have

$$
V_{k}=l_{k 1} \operatorname{div} u^{p}+\sum_{j=2}^{4} l_{k j} \partial_{t} u_{j-1}^{p}+l_{k 5} \theta+\sum_{j=6}^{8} l_{k j} q_{j-5}^{p}, \quad 1 \leq k \leq 8,
$$

which gives

$$
\begin{aligned}
V_{k}= & \left(\frac{1}{2}+\mathcal{O}\left(\tau^{2}\right)\right)\left[-\frac{\alpha^{2} \delta \tau}{\kappa \gamma}(1+\mathcal{O}(\tau)) \operatorname{div} u^{p} \pm \delta \sqrt{\frac{\tau}{\kappa \gamma}}(1+\mathcal{O}(\tau)) \nabla \Phi^{0} \cdot \partial_{t} u^{p}\right. \\
& \left.+\theta \pm \sqrt{\frac{\tau \gamma}{\kappa}}(1+\mathcal{O}(\tau)) \nabla \Phi^{0} \cdot q^{p}\right]
\end{aligned}
$$

for $k=5,6$, and

$$
\begin{aligned}
V_{k}= & \left(\frac{\alpha^{2} \beta \delta}{2 \kappa^{2} \gamma^{2}} \tau^{2}+\mathcal{O}\left(\tau^{3}\right)\right)\left[\frac{\kappa \gamma}{\beta \tau}(1+\mathcal{O}(\tau)) \operatorname{div} u^{p} \mp \frac{\kappa \gamma}{\alpha \beta \tau}(1+\mathcal{O}(\tau)) \nabla \Phi^{0} \cdot \partial_{t} u^{p}\right. \\
& \left.+\theta \pm \frac{\gamma}{\alpha}(1+\mathcal{O}(\tau)) \nabla \Phi^{0} \cdot q^{p}\right]
\end{aligned}
$$

for $k=7,8$.

Suppose that

$$
\left[\operatorname{div} u^{0, p}\right]_{\sigma}, \quad\left[u^{1, p}\right]_{\sigma}, \quad\left[\theta^{0}\right]_{\sigma}, \quad\left[q^{0, p}\right]_{\sigma}
$$


may not vanish for the initial data given in (1.4). Then from (3.67), (3.68) we have

$$
\left[V_{k}^{0}\right]_{\sigma}=\left\{\begin{array}{c}
-\frac{\alpha^{2} \delta \tau}{2 \kappa \gamma}(1+\mathcal{O}(\tau))\left[\operatorname{div} u^{0, p}\right]_{\sigma} \pm \frac{\delta}{2} \sqrt{\frac{\tau}{\kappa \gamma}}(1+\mathcal{O}(\tau)) \nabla \Phi^{0} \cdot\left[u^{1, p}\right]_{\sigma} \\
+\left(\frac{1}{2}+\mathcal{O}(\tau)\right)\left[\theta^{0}\right]_{\sigma} \pm \frac{1}{2} \sqrt{\frac{\tau \gamma}{\kappa}}(1+\mathcal{O}(\tau)) \nabla \Phi^{0} \cdot\left[q^{0, p}\right]_{\sigma}, \quad k=5,6 \\
\frac{\alpha^{2} \delta \tau}{2 \kappa \gamma}(1+\mathcal{O}(\tau))\left[\operatorname{div} u^{0, p}\right]_{\sigma} \mp \frac{\alpha \delta \tau}{2 \kappa \gamma}(1+\mathcal{O}(\tau)) \nabla \Phi^{0} \cdot\left[u^{1, p}\right]_{\sigma} \\
+\frac{\alpha^{2} \beta \delta}{2 \kappa^{2} \gamma^{2}} \tau^{2}(1+\mathcal{O}(\tau))\left[\theta^{0}\right]_{\sigma} \pm \frac{\alpha \beta \gamma}{2 \kappa^{2} \gamma} \tau^{2}(1+\mathcal{O}(\tau)) \nabla \Phi^{0} \cdot\left[q^{0, p}\right]_{\sigma}, \quad k=7,8
\end{array}\right.
$$

Substituting (3.69) into (3.63)-(3.66), it follows that

$$
\begin{aligned}
& {\left[\operatorname{div} u^{p}\right]_{\Sigma_{k}}=}\left\{\begin{array}{c}
\mathcal{O}(\tau) e^{-\frac{t}{2 \tau}(1+\mathcal{O}(\sqrt{\tau}))}, \quad k=5,6, \\
\frac{1}{2 \alpha}\left(\alpha\left[\operatorname{div} u^{0, p}\right]_{\sigma} \mp \nabla \Phi^{0} \cdot\left[u^{1, p}\right]_{\sigma}+\mathcal{O}(\tau)\right) . \\
\cdot e^{-\int_{0}^{t}\left(\frac{\beta \delta}{2 \kappa \gamma} \pm \frac{\alpha}{2} \Delta \Phi^{0}\left(x\left(s ; 0, x^{0}\right)\right)+\mathcal{O}(\tau)\right) d s}, \quad k=7,8,
\end{array}\right. \\
& {\left[u_{t}^{p}\right]_{\Sigma_{k}}=\left\{\begin{array}{c}
\mathcal{O}(\sqrt{\tau}) e^{-\frac{t}{2 \tau}(1+\mathcal{O}(\sqrt{\tau}))}, \quad k=5,6, \\
\mp \frac{1}{2}\left(\alpha\left[\operatorname{div} u^{0, p}\right]_{\sigma} \mp \nabla \Phi^{0} \cdot\left[u^{1, p}\right]_{\sigma}+\mathcal{O}(\tau)\right) . \\
\cdot e^{-\int_{0}^{t}\left(\frac{\beta \delta}{2 \kappa \gamma} \pm \frac{\alpha}{2} \Delta \Phi^{0}\left(x\left(s ; 0, x^{0}\right)\right)+\mathcal{O}(\tau)\right) d s}, \quad k=7,8,
\end{array}\right.} \\
& {[\theta]_{\Sigma_{k}}=\left\{\begin{array}{c}
\frac{1}{2}\left(\left[\theta^{0}\right]_{\sigma}+\mathcal{O}(\sqrt{\tau})\right) e^{-\frac{t}{2 \tau}(1+\mathcal{O}(\sqrt{\tau}))}, \quad k=5,6, \\
\mathcal{O}(\tau) e^{-\int_{0}^{t}\left(\frac{\beta \delta}{2 \kappa \gamma} \pm \frac{\alpha}{2} \Delta \Phi^{0}\left(x\left(s ; 0, x^{0}\right)\right)+\mathcal{O}(\tau)\right) d s}, \quad k=7,8,
\end{array}\right.} \\
& {\left[q^{p}\right]_{\Sigma_{k}}=\left\{\begin{array}{c}
\left( \pm \frac{1}{2} \sqrt{\frac{\kappa}{\gamma \tau}}\left[\theta^{0}\right]_{\sigma}+\frac{1}{2} \nabla \Phi^{0}\left(\left[q^{0, p}+\frac{\delta}{\gamma} u^{1, p}\right]_{\sigma} \cdot \nabla \Phi^{0}\right)+\mathcal{O}(\sqrt{\tau})\right) e^{-\frac{t}{2 \tau}(1+\mathcal{O}(\sqrt{\tau}))}, k=5,6, \\
\pm \frac{\delta}{2 \gamma}\left(\left(\alpha\left[\operatorname{div} u^{0, p}\right]_{\sigma} \mp \nabla \Phi^{0} \cdot\left[u^{1, p}\right]_{\sigma}\right) \nabla \Phi^{0}+\mathcal{O}(\tau)\right) . \\
\cdot e^{-\int_{0}^{t}\left(\frac{\beta \delta}{2 \kappa \gamma} \pm \frac{\alpha}{2} \Delta \Phi^{0}\left(x\left(s ; 0, x^{0}\right)\right)+\mathcal{O}(\tau)\right) d s}, k=7,8 .
\end{array}\right.}
\end{aligned}
$$

Taking the above discussion altogether we conclude

TheOREm 3.3. Suppose that the initial data of $\operatorname{div} u^{p}, \partial_{t} u^{p}, \theta$ and $q^{p}$ may have jumps on $\sigma=\left\{\Phi^{0}(x)=0\right\}$ with $\left|\nabla \Phi^{0}(x)\right|=1$. Then the propagation of strong singularities of solutions to the linearized problem (2.11)-(2.14) $\left(f^{p}=g=0\right)$ is described by (3.70)(3.73). In particular, we have

(1) The jumps of $\operatorname{div} u^{p}, \partial_{t} u^{p}, \theta, q^{p}$ on $\Sigma_{5}$ and $\Sigma_{6}$ decay exponentially both when $\tau \rightarrow 0$ for a fixed $t>0$ and when $t \rightarrow+\infty$ for a fixed $\tau>0$.

(2) The jumps of $\operatorname{div} u^{p}, \partial_{t} u^{p}, q^{p}$ on $\Sigma_{7}$ (resp. $\Sigma_{8}$ ) are propagated, and when $t \rightarrow$ $+\infty$ they will decay exponentially as soon as $\frac{\beta \delta}{\kappa \gamma}+\alpha \Delta \Phi^{0}$ (resp. $\frac{\beta \delta}{\kappa \gamma}-\alpha \Delta \Phi^{0}$ ) becomes positive, more rapidly for the smaller heat conductive coefficient $\kappa \gamma$, while the jump of the temperature $\theta$ on $\Sigma_{7}$ and $\Sigma_{8}$ vanishes of order $O(\tau)$ when $\tau \rightarrow 0$, which shows a smoothing effect in the system (2.11) - 2.13) when the thermoelastic model with second sound converges to the hyperbolic-parabolic type of classical thermoelasticity. 
4. A semilinear problem. In this section, we shall mainly study the asymptotic behavior of discontinuities in the following semilinear problem:

$$
\begin{aligned}
u_{t t}-\alpha^{2} \Delta u+\beta \nabla \theta & =f\left(u_{t}, \theta\right), \\
\theta_{t}+\gamma \operatorname{div} q+\delta \operatorname{div} u_{t} & =g\left(u_{t}, \theta\right), \\
\tau q_{t}+q+\kappa \nabla \theta & =0,
\end{aligned}
$$

on $(0, \infty) \times \mathbb{R}^{3}$,

$$
u(t=0)=u^{0}, u_{t}(t=0)=u^{1}, \theta(t=0)=\theta^{0}, q(t=0)=q^{0}
$$

with all coefficients being the same as in (2.11)-(2.13), and

$$
\text { curl } u=0, \quad \operatorname{curl} q^{0}=0 .
$$

By comparing the above problem with the problem (2.1)-(2.4), it is easy to see that if we assume that the nonlinear function $f$ in (2.1) is a conserved field, i.e. there is a scalar function $\tilde{g}$ such that $f=\nabla \tilde{g}$, then from the problems (2.7), (2.8) and (2.9), (2.10), we know that the solenoidal part $\left(u^{s}, q^{s}\right)$ vanishes when the initial data satisfy curl $u^{0}=\operatorname{curl} u^{1}=0$. Therefore, we get that the unknowns $(u, \theta, q)$ to the original thermoelastic systems (2.1)-(2.3) of second sound satisfy the above problem. In the remainder, we shall only focus on the problem (4.1)-(4.5).

Denote $U=\left(\operatorname{div} u, u_{t}, \theta, q\right)^{\prime}$, and let $L=\left(l_{1}^{\prime}, \ldots, l_{8}^{\prime}\right)^{\prime}$ be the matrix composed of the left eigenvectors $\left\{l_{j}\right\}_{j=1}^{8}$ given in (3.17)-(3.19). As in $\S 3$, we know that $V=L U$ satisfies the problem (3.29), (3.30):

$$
\begin{gathered}
\partial_{t} V+\sum_{j=1}^{3}\left(L A_{j} R\right) \partial_{j} V+\widetilde{A}_{0} V=\widetilde{F}, \\
V(t=0)=V^{0}:=L U^{0},
\end{gathered}
$$

where $\tilde{F}(V)=L \cdot(0, f(V), g(V), 0)^{\prime}$, with

$$
f(V)=f\left(\sum_{k=1}^{8} r_{2 k} V_{k}, \ldots, \sum_{k=1}^{8} r_{5 k} V_{k}\right)
$$

and $g(V)$ having the same form.

Obviously, the results stated in Lemma 3.1 still hold for the problem (4.6), (4.7). Thus, $\left[V_{j}\right]_{\Sigma_{k}}=0$ for $1 \leq j \leq 4,5 \leq k \leq 8$, and $5 \leq j \leq 8,1 \leq k \leq 8$ with $j \neq k$, and the jump of $V_{k}$ on $\Sigma_{k}$ satisfies the transport equation:

$$
\left(\partial_{t}+\sum_{j=1}^{3}\left(L A_{j} R\right)_{k k} \partial_{x_{j}}+\left(\widetilde{A}_{0}\right)_{k k}\right)\left[V_{k}\right]_{\Sigma_{k}}=\left[\tilde{F}_{k}\right]_{\Sigma_{k}},
$$

where

$$
\tilde{F}_{k}(V)=\sum_{j=2}^{4} l_{k j} f_{j}(V)+l_{k 5} g(V)
$$


Suppose that $f$ and $g$ are globally Lipschitz in their arguments. From (3.16), (3.19) we have

$$
\begin{aligned}
\left|\left[\tilde{F}_{k}\right]_{\Sigma_{k}}\right| \leq & \sum_{l=1}^{4}\left\{\sum_{j=1}^{3}\left|l_{k(j+1)}\right| \cdot\left\|\partial_{l} f_{j}\right\|_{L^{\infty}}+\left|l_{k 5}\right| \cdot\left\|\partial_{l} g\right\|_{L^{\infty}}\right\}\left|r_{(l+1) k}\right| \cdot\left|\left[V_{k}\right]_{\Sigma_{k}}\right| \\
\leq & \left|c_{k}\right|\left\{\sum_{l=1}^{3}\left|\frac{\beta \lambda_{k}}{\alpha^{2}-\lambda_{k}^{2}} \partial_{x_{l}} \Phi^{0}\right|\left(\sum_{j=1}^{3}\left|\frac{\delta \lambda_{k}}{\alpha^{2}-\lambda_{k}^{2}} \partial_{x_{j}} \Phi^{0}\right| \cdot\left\|\partial_{l} f_{j}\right\|_{L^{\infty}}+\left\|\partial_{l} g\right\|_{L^{\infty}}\right)\right. \\
& \left.+\sum_{j=1}^{3}\left|\frac{\delta \lambda_{k}}{\alpha^{2}-\lambda_{k}^{2}} \partial_{x_{j}} \Phi^{0}\right| \cdot\left\|\partial_{4} f_{j}\right\|_{L^{\infty}}+\left\|\partial_{4} g\right\|_{L^{\infty}}\right\}\left|\left[V_{k}\right]_{\Sigma_{k}}\right| .
\end{aligned}
$$

By using (3.61), (3.62), we have

$$
\left|\left[\tilde{F}_{k}(V)\right]_{\Sigma_{k}}\right| \leq\left\{\begin{array}{l}
\mathcal{O}(1)\left|\left[V_{k}\right]_{\Sigma_{k}}\right|, \quad k=5,6, \\
\left(\frac{1}{2} \sum_{j, l=1}^{3}\left|\partial_{x_{j}} \Phi^{0} \partial_{x_{l}} \Phi^{0}\right||| \partial_{l} f_{j} \|_{L^{\infty}}+\mathcal{O}(\tau)\right)\left|\left[V_{k}\right]_{\Sigma_{k}}\right|, \quad k=7,8 .
\end{array}\right.
$$

As in (3.50), for any fixed $x^{0} \in \sigma$, denote by

$$
t \rightarrow\left(t, x_{1}\left(t ; 0, x^{0}\right), x_{2}\left(t ; 0, x^{0}\right), x_{3}\left(t ; 0, x^{0}\right)\right)
$$

the characteristic line of the operator $\partial_{t}+\sum_{j=1}^{3}\left(L A_{j} R\right)_{k k} \partial_{x_{j}}$ passing through $\left(0, x^{0}\right)$, and

$$
\left[V_{k}\right]_{\Sigma_{k}(t)}=\left[V_{k}\right]_{\Sigma_{k}}\left(t, x_{1}\left(t ; 0, x^{0}\right), x_{2}\left(t ; 0, x^{0}\right), x_{3}\left(t ; 0, x^{0}\right)\right) .
$$

Then, from (4.8) we have

$$
\partial_{t}\left[V_{k}\right]_{\Sigma_{k}(t)}+\left(\widetilde{A}_{0}\right)_{k k}\left(x\left(t ; 0, x^{0}\right)\right)\left[V_{k}\right]_{\Sigma_{k}(t)}=\left[\tilde{F}_{k}\right]_{\Sigma_{k}(t)} .
$$

From (4.10) we immediately obtain

$$
\left[V_{k}\right]_{\Sigma_{k}(t)}=\left[V_{k}\right]_{\Sigma_{k}(0)} e^{-\int_{0}^{t}\left(\tilde{A_{0}}\right)_{k k}\left(x\left(s ; 0, x^{0}\right)\right) d s}+\int_{0}^{t}\left[\tilde{F}_{k}\right]_{\Sigma_{k}(s)} e^{-\int_{s}^{t}\left(\tilde{A_{0}}\right)_{k k}\left(x\left(s_{1} ; 0, x^{0}\right)\right) d s_{1}} d s .
$$

From (4.11) and (4.9), we see that for $k=5,6$, the behavior of $\left[V_{k}\right]_{\Sigma_{k}}$ is the same as that given in (3.51).

When $k=7,8$, from (4.11) and (4.9), we have

$\left|\left[V_{k}\right]_{\Sigma_{k}(t)}\right|$

$$
\leq\left|\left[V_{k}\right]_{\Sigma_{k}(0)}\right| \exp \left(-\int_{0}^{t}\left(\tilde{A}_{0}\right)_{k k}\left(x\left(s ; 0, x^{0}\right)\right) d s+\left(\frac{1}{2} \sum_{j, l=1}^{3}\left\|\partial_{l} f_{j}\right\|_{L^{\infty}}\left\|\partial_{x_{j}} \Phi^{0} \partial_{x_{l}} \Phi^{0}\right\|_{L^{\infty}}+\mathcal{O}(\tau)\right) t\right)
$$

and

$$
\begin{aligned}
& \left|\left[V_{k}\right]_{\Sigma_{k}(t)}\right| \geq\left|\left[V_{k}\right]_{\Sigma_{k}(0)}\right| \exp \left(-\int_{0}^{t}\left(\tilde{A}_{0}\right)_{k k}\left(x\left(s ; 0, x^{0}\right)\right) d s\right) \\
& -\int_{0}^{t}\left(\frac{1}{2} \sum_{j, l=1}^{3}\left\|\partial_{l} f_{j}\right\|_{L^{\infty}}\left\|\partial_{x_{j}} \Phi^{0} \partial_{x_{l}} \Phi^{0}\right\|_{L^{\infty}}+\mathcal{O}(\tau)\right)\left|\left[V_{k}\right]_{\Sigma_{k}(s)}\right| e^{-\int_{s}^{t}\left(\tilde{\left.A_{0}\right)_{k k}}\left(x\left(s_{1} ; 0, x^{0}\right)\right) d s_{1}\right.} d s .
\end{aligned}
$$

By using the estimate (4.12) for $\left[V_{k}\right]_{\Sigma_{k}(s)}$ in the second term on the right-hand side of (4.13), one gets

$\left|\left[V_{k}\right]_{\Sigma_{k}(t)}\right| \geq\left|\left[V_{k}\right]_{\Sigma_{k}(0)}\right| e^{-\int_{0}^{t}\left(\tilde{A_{0}}\right)_{k k}\left(x\left(s ; 0, x^{0}\right)\right) d s}\left(2-e^{\left(\frac{1}{2} \sum_{j, l=1}^{3}\left\|\partial_{l} f_{j}\right\|_{L} \infty\left\|\partial_{x_{j}} \Phi^{0} \partial_{x_{l}} \Phi^{0}\right\|_{L}+\mathcal{O}(\tau)\right) t}\right)$. 
To obtain the information of $U=\left(\operatorname{div} u, u_{t}, \theta, q\right)^{\prime}$, we follow the discussion from (3.53) to (3.73), and deduce that the jumps of $\left(\operatorname{div} u, u_{t}, \theta, q\right)$ on $\Sigma_{5}$ and $\Sigma_{6}$ have the same behaviour as in (3.70) $-(3.73)$, and on $\Sigma_{k}$ for $k=7,8$,

$$
\begin{aligned}
& \left\{\begin{aligned}
&\left|[\operatorname{div} u]_{\Sigma_{k}(t)}\right| \leq \frac{1}{2 \alpha}\left|\alpha\left[\operatorname{div} u^{0}\right]_{\sigma} \mp \nabla \Phi^{0} \cdot\left[u^{1}\right]_{\sigma}+\mathcal{O}(\tau)\right| \\
& \cdot e^{-\frac{\beta \delta}{2 \kappa \gamma} t+\left(\frac{1}{2} \sum_{j, l=1}^{3}\left\|\partial_{l} f_{j}\right\|_{L} \infty\left\|\partial_{x_{j}} \Phi^{0} \partial_{x_{l}} \Phi^{0}\right\|_{L} \infty+\mathcal{O}(\tau)\right) t \mp \frac{\alpha}{2} \int_{0}^{t} \Delta \Phi^{0}\left(x\left(s ; 0, x^{0}\right)\right) d s}, \\
&\left|[\operatorname{div} u]_{\Sigma_{k}(t)}\right| \geq \frac{1}{2 \alpha}\left|\alpha\left[\operatorname{div} u^{0}\right]_{\sigma} \mp \nabla \Phi^{0} \cdot\left[u^{1}\right]_{\sigma}+\mathcal{O}(\tau)\right| \\
& \cdot e^{-\frac{\beta \delta}{2 \kappa \gamma} t \mp \frac{\alpha}{2} \int_{0}^{t}\left(\Delta \Phi^{0}\left(x\left(s ; 0, x^{0}\right)\right)+\mathcal{O}(\tau)\right) d s}\left(2-e^{\left(\frac{1}{2} \sum_{j, l=1}^{3}\left\|\partial_{l} f_{j}\right\|_{L} \infty\left\|\partial_{x_{j}} \Phi^{0} \partial_{x_{l}} \Phi^{0}\right\|_{L} \infty+\mathcal{O}(\tau)\right) t}\right),
\end{aligned}\right.
\end{aligned}
$$

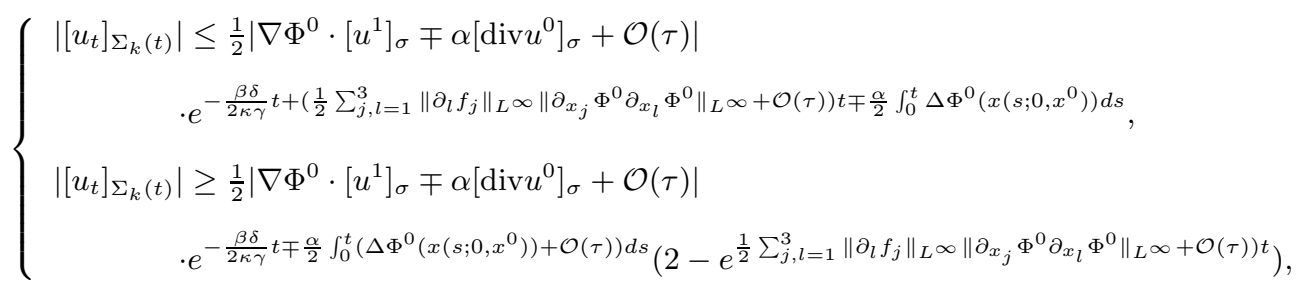

$$
\begin{aligned}
& \left|[\theta]_{\Sigma_{k}(t)}\right| \leq \mathcal{O}(\tau) e^{-\frac{\beta \delta}{2 \kappa \gamma} t+\left(\frac{1}{2} \sum_{j, l=1}^{3}\left\|\partial_{l} f_{j}\right\|_{L} \infty\left\|\partial_{x_{j}} \Phi^{0} \partial_{x_{l}} \Phi^{0}\right\|_{L} \infty+\mathcal{O}(\tau)\right) t \mp \frac{\alpha}{2} \int_{0}^{t} \Delta \Phi^{0}\left(x\left(s ; 0, x^{0}\right)\right) d s}, \\
& \left\{\begin{aligned}
\left|[q]_{\Sigma_{k}(t)}\right| & \leq \frac{\delta}{2 \gamma}\left|\left(\nabla \Phi^{0} \cdot\left[u^{1}\right]_{\sigma} \mp \alpha\left[\operatorname{div} u^{0}\right]_{\sigma}\right) \nabla \Phi^{0}+\mathcal{O}(\tau)\right| \\
& \cdot e^{-\frac{\beta \delta}{2 \kappa \gamma} t+\left(\frac{1}{2} \sum_{j, l=1}^{3}\left\|\partial_{l} f_{j}\right\|_{L} \infty\left\|\partial_{x_{j}} \Phi^{0} \partial_{x_{l}} \Phi^{0}\right\|_{L} \infty+\mathcal{O}(\tau)\right) t \mp \frac{\alpha}{2} \int_{0}^{t} \Delta \Phi^{0}\left(x\left(s ; 0, x^{0}\right)\right) d s}, \\
\left|[q]_{\Sigma_{k}(t)}\right| & \geq \frac{\delta}{2 \gamma}\left|\left(\nabla \Phi^{0} \cdot\left[u^{1}\right]_{\sigma} \mp \alpha\left[\operatorname{div} u^{0}\right]_{\sigma}\right) \nabla \Phi^{0}+\mathcal{O}(\tau)\right| \\
& \cdot e^{-\frac{\beta \delta}{2 \kappa \gamma} t \mp \frac{\alpha}{2} \int_{0}^{t}\left(\Delta \Phi^{0}\left(x\left(s ; 0, x^{0}\right)\right)+\mathcal{O}(\tau)\right) d s}\left(2-e^{\left.\frac{1}{2} \sum_{j, l=1}^{3}\left\|\partial_{l} f_{j}\right\|_{L} \infty\left\|\partial_{x_{j}} \Phi^{0} \partial_{x_{l}} \Phi^{0}\right\|_{L} \infty+\mathcal{O}(\tau)\right) t}\right) .
\end{aligned}\right.
\end{aligned}
$$

Therefore, similar to Theorem 4.1 of [11, we conclude

Theorem 4.1. For the problem (4.1)-(4.5), in addition to the assumptions of Theorem 3.3, we also suppose that the nonlinear functions $f$ and $g$ are smooth and globally Lipschitz in their arguments. Then, we have

(1) The jumps of $\operatorname{div} u, \partial_{t} u, \theta, q$ on $\Sigma_{5}$ and $\Sigma_{6}$ decay exponentially both when $\tau \rightarrow 0$ for a fixed $t>0$ and when $t \rightarrow+\infty$ for a fixed $\tau>0$.

(2) The jumps of $\operatorname{div} u, \partial_{t} u, q$ on $\Sigma_{7}\left(\right.$ resp. $\Sigma_{8}$ ) are propagated, and when $t \rightarrow+\infty$ they will decay exponentially as soon as

$$
\begin{gathered}
\frac{\beta \delta}{\kappa \gamma}+\alpha \Delta \Phi^{0}-\sum_{j, l=1}^{3}\left\|\partial_{l} f_{j}\right\|_{L^{\infty}}\left\|\partial_{x_{j}} \Phi^{0} \partial_{x_{l}} \Phi^{0}\right\|_{L^{\infty}}, \\
\left(\operatorname{resp} \cdot \frac{\beta \delta}{\kappa \gamma}-\alpha \Delta \Phi^{0}-\sum_{j, l=1}^{3}\left\|\partial_{l} f_{j}\right\|_{L^{\infty}}\left\|\partial_{x_{j}} \Phi^{0} \partial_{x_{l}} \Phi^{0}\right\|_{L^{\infty}}\right)
\end{gathered}
$$

is positive, more rapidly for the smaller heat conductive coefficient $\kappa \gamma$, while the jump of the temperature $\theta$ on $\Sigma_{7}$ and $\Sigma_{8}$ vanishes of order $O(\tau)$ when $\tau \rightarrow 0$. 
REMARK 4.1. It is easy to carry out the above discussion as well for the case that the nonlinear functions $f$ and $g$ depend on $\operatorname{div} u$ and $q$ in the problem (4.1)-(4.5).

Acknowledgments. The authors were supported by the Chinese-German NSF/ DFG-project "Partial Differential Equations and Applications in Geometry and Physics", DFG-GZ: 446 CHV 113/170/0-Z. The work of Ya-Guang Wang is also partially supported by a key project from the NSFC of China under the grant 10531020 and a joint project from the NSAF of China under the grant 10676020.

\section{REFERENCES}

[1] Beals, M.: Propagation and Interaction of Singularities in Nonlinear Hyperbolic Problems. Birkhäuser, Boston, 1989. MR1033737(91a:58183)

[2] Chandrasekharaiah, D. S.: Hyperbolic thermoelasticity: A review of recent literature. Appl. Mech. Rev. 51 (1998), 705-729.

[3] Chen, S. and Wang, Y. G.: Propagation of singularities of solutions to hyperbolic-parabolic coupled systems. Math. Nachr., 242(2002), 46-60. MR1916849 (2003e:35008)

[4] Hoff, D.: Dynamics of singularity surfaces for compressible, viscous flows in two space dimensions. Commun. Pure Appl. Math. 55 (2002), 1365-1407. MR.1916987(2003k:76108)

[5] Gilbarg, D. and Trudinger, N.S.: Elliptic Partial Differential Equations of Second Order, Grundlehren math. Wiss. 224, Springer-Verlag, Berlin (1983). MR737190 (86c:35035)

[6] Jiang, S. and Racke, R.: Evolution Equations in Thermoelasticity, Monographs and Surveys in Pure Appl. Math. 112, Chapman \& Hall/CRC, Boca Raton (2000). MR.1774100 (2001g:74013)

[7] Métivier, G.: Propagation, interaction and reflection of discontinuous progressing waves for semilinear hyperbolic systems. Amer. J. Math. 111 (1989), 239-287. MR.987758 (90g:35097)

[8] Racke, R.: Thermoelasticity with second sound - exponential stability in linear and non-linear 1-d. Math. Meth. Appl. Sci. 25 (2002), 409-441. MR.1888164 (2002m:74016)

[9] Racke, R.: Asymptotic behavior of solutions in linear 2- or 3-d thermoelasticity with second sound. Quart. Appl. Math. 61 (2003), 315-328. MR1976372(2004a:74026)

[10] Racke, R. and Wang, Y. G.: Propagation of singularities in one-dimensional thermoelasticity. $J$. Math. Anal. Appl. 223 (1998), 216-247. MR.1627324 (99f:73014)

[11] Racke, R. and Wang, Y. G.: Asymptotic behavior of discontinuous solutions to thermoelastic systems with second sound. J. Anal. Appl. 24 (2005), 117-135. MR2146553 (2006k:74040)

[12] Rauch, J. and Reed, M.: Discontinuous progressing waves for semilinear systems. Commun. PDE 10 (1985), 1033-1075. MR806255 (87g:35146)

[13] Wang, Y.G.: Microlocal analysis in nonlinear thermoelasticity. Nonlinear Anal. 54 (2003), 683705. MR 1983443(2004b:74022)

[14] Wang, Y.G.: A new approach to study hyperbolic-parabolic coupled systems. In "Evolution Equations" (R. Picard, M. Reissig \& W. Zajaczkowski, eds.), Banach Center Publications, 60(2003), 227-236. MR 1993072 (2004h:35164) 